\title{
Multilevel Hierarchical Topographies by Combined Photolithography and Nanoimprinting Processes to Create Surfaces with Controlled Wetting
}

María T. Alameda, Manuel R. Osorio, Jaime J. Hernández* and Isabel Rodríguez

Madrid Institute for Advanced Studies in Nanoscience (IMDEA Nanoscience)

C/Faraday 9, Ciudad Universitaria de Cantoblanco, Madrid 28049, Spain.

*Corresponding Author.

Email: jaime.hernandez@imdea.org

Figure S1 shows a two-level, third order, hierarchical surface fabricated using photomask lithography. To fabricate this topography, first a bottom layer of polycarbonate was thermally imprinted with $2 \mu \mathrm{m}$ diameter pore array. Over this, a Epo-Tek film was casted and imprinted with $2 \mu \mathrm{m}$ pillar patterns using a transparent mold while kept at $60^{\circ} \mathrm{C}$ to facilitate the filling of the structures before UV exposure is performed trough a lithographic mask of microchannels patterns. An enlarged SEM image of the $2 \mu \mathrm{m}$ micropores and pillars on top and bottom of the channels is also revealed.

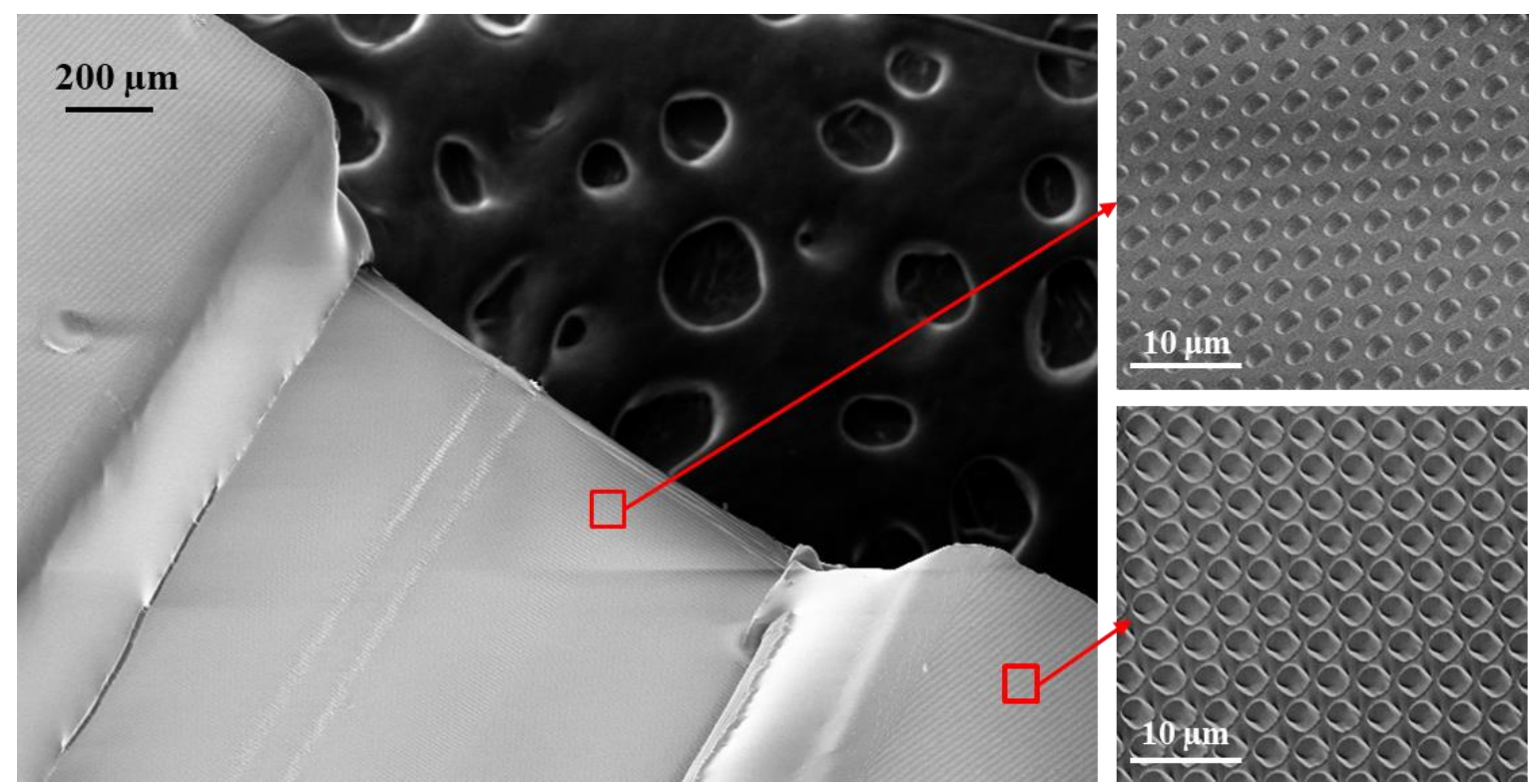

Figure S1. SEM images of a two-level, third order hierarchical surface fabricated by traditional mask photolithography. (The black perforated area is the carbon tape used for fixing the sample during SEM). 
Figure S2 shows SEM images at different replication stages of a two-level second-order hierarchical structure fabrication process and materials employed during the sequential replication steps.
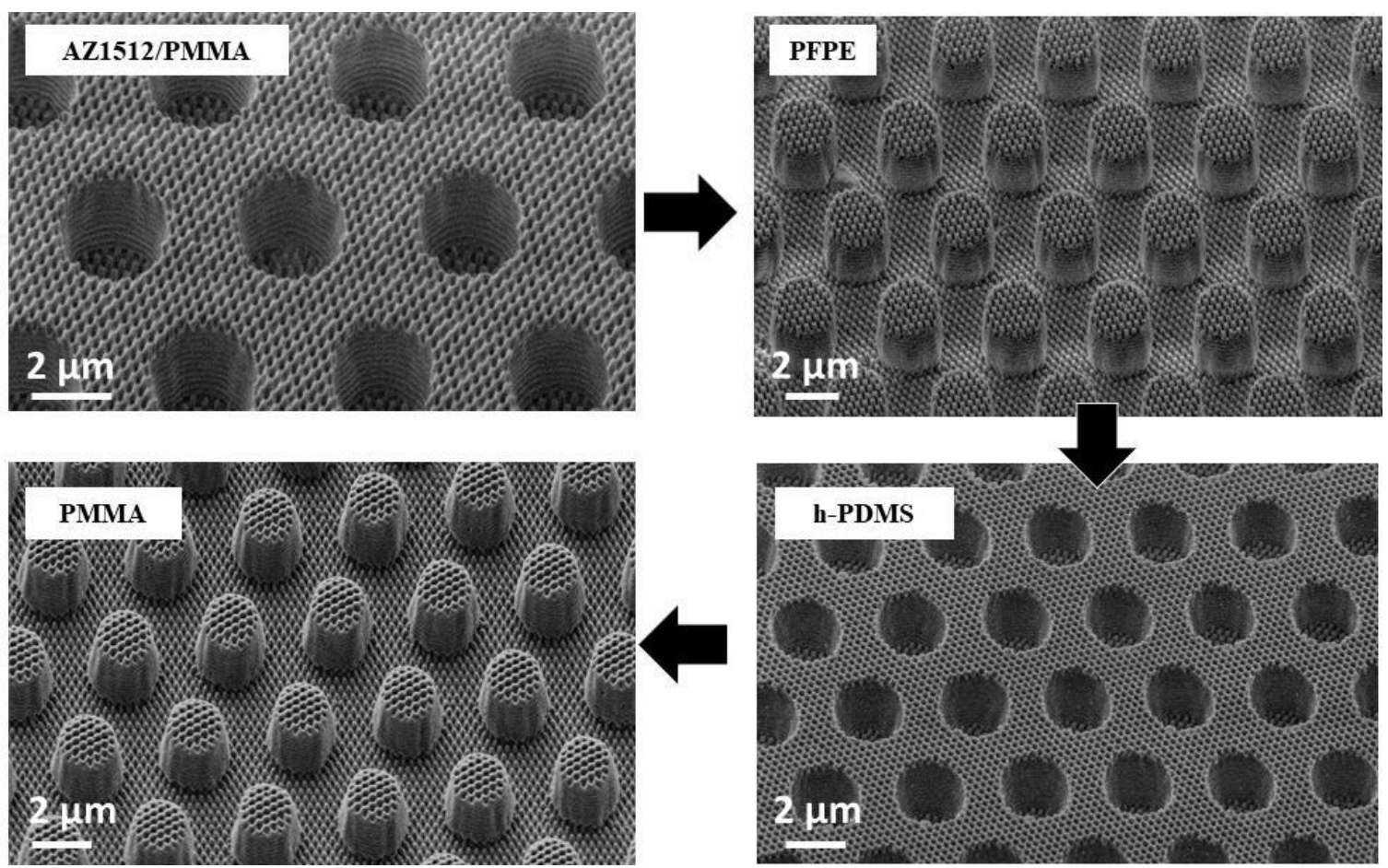

Figure S2. SEM images of the hierarchical surfaces at different replication stages from the hierarchical master substrate to the h-PDMS working mold and the final PMMA imprinted hierarchical topography. 
Figure S3 shows SEM images of PMMA texturized films used for the wetting characterization.
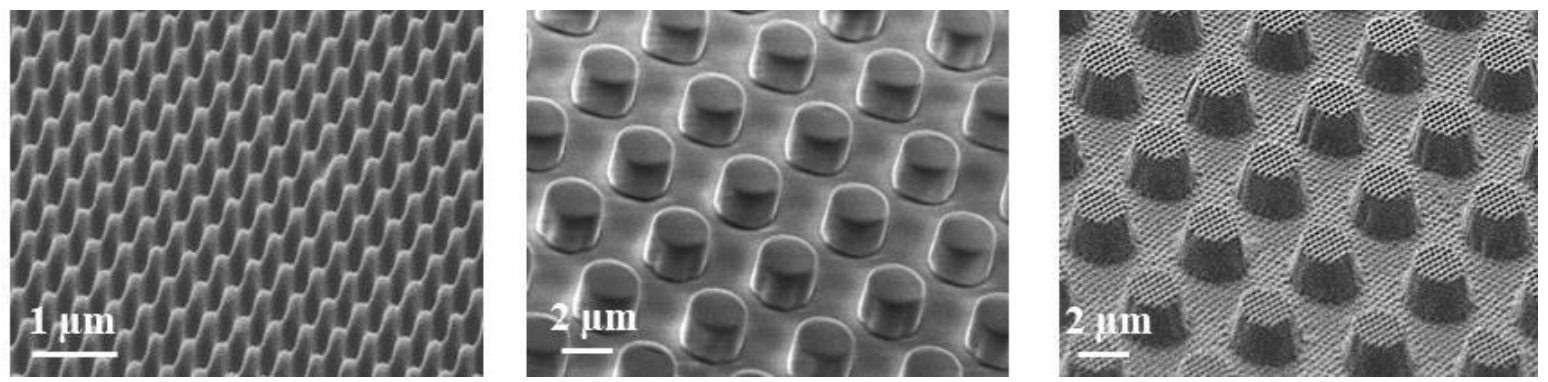

Figure S3. SEM images of moth-eye nanocone topography (left), $2 \times 2 \mu \mathrm{m}$ pillar array (middle) and the corresponding two-level second order hierarchical topography (right) fabricated on PMMA films.

Figure S4 shows micrographs of water droplets on a flat surface, on a $2 \times 2 \mu \mathrm{m}$ pillar microstructured surface, on a moth-eye nanocone surface and a hierarchical topography imprinted on different materials (PDMS, PFPE and PMMA).

The meniscus shape fitting for the water contact angle (WCA) calculation is included in each case. A superhydrophobic character $\left(\mathrm{WCA} \geq 150^{\circ}\right)$ arises in all materials upon nanoimprinting the hierarchical topography.
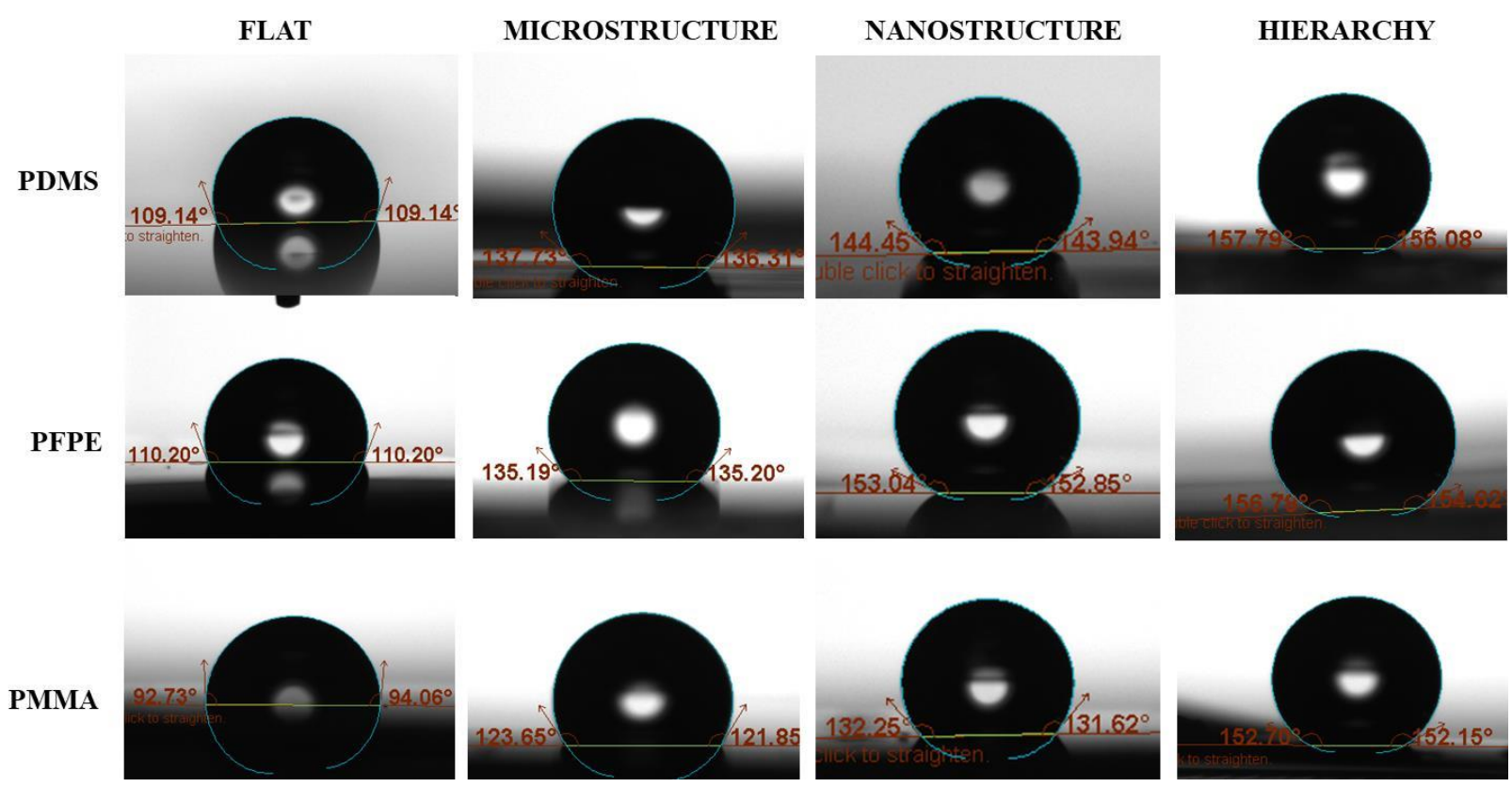

Figure S4. Images of $c a .4 \mu \mathrm{l}$ drops of water deposited on the different films surfaces. Fitting for the WCA calculation is shown in each case. 
Figure S5 shows the WCA of a water droplet deposited over a flat film of AZ1512 photoresist.

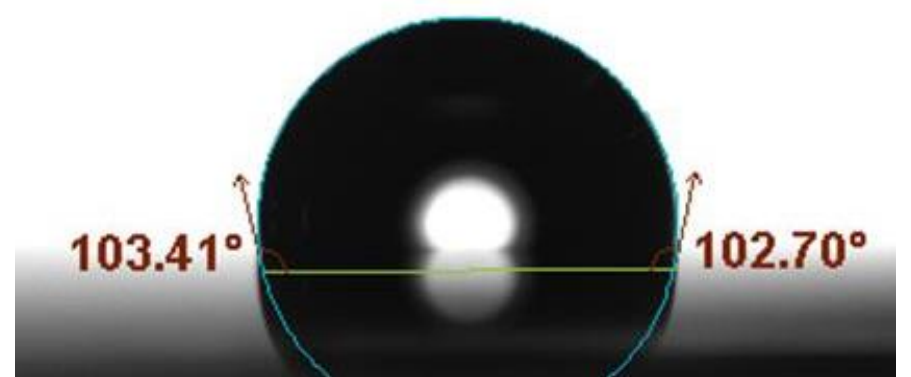

Figure S5. Images of a $4 \mu$ drop of water deposited on a flat AZ1512 resist film. WCA calculation fitting is included.

Figure S6 illustrates the angular dependence of the moth-eye topography appearance during SEM imaging. A moth-eye patterned polymer substrate was fixed at a tilting angle and then rotated. Images were acquired at different rotation degrees.
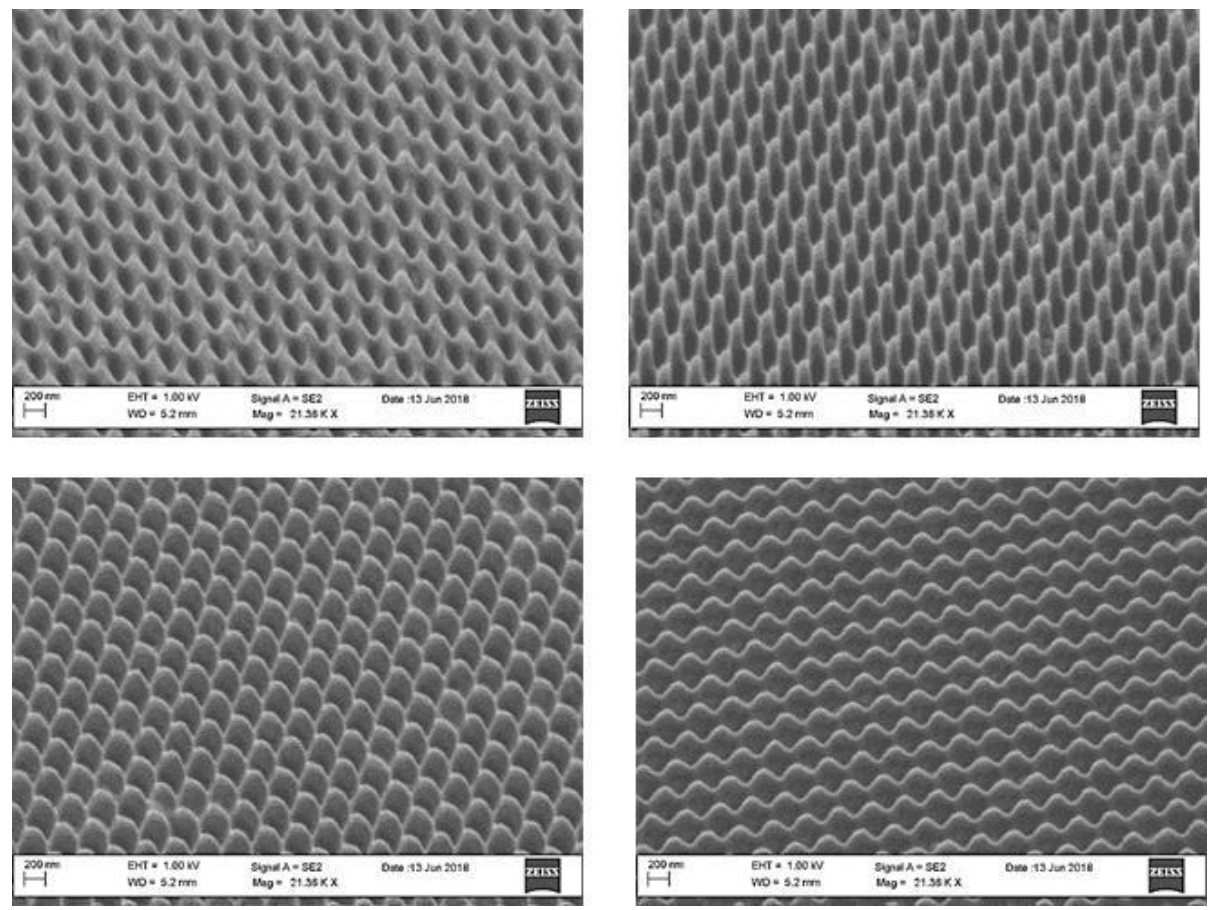

Figure S6. SEM images of a nanostructured PMMA surface obtained for a fixed tilting and different rotation angles. 